\title{
Taking a stab at neuronal heterogeneity
}

Patch-seq integrates functional and molecular properties of single neurons to tackle heterogeneity in the brain.

Neurons are usually identified by either their molecular profile or their electrophysiological and morphological properties. Combining these features would be informative for characterization of neuronal diversity.

Two teams have independently reported developing the Patch-seq technology by combining whole-cell patch-clamp recordings with subsequent RNA-seq of individual neurons. Sten Linnarsson, Tibor Harkany and their colleagues at Karolinska Institutet in Stockholm and the Medical University of Vienna used Patch-seq to characterize neurons in the mouse somatosensory cortex (Fuzik et al., 2015). Rickard Sandberg, Andreas Tolias and their coworkers at Baylor College of Medicine in Houston and Karolinska Institutet assessed neocortical diversity in the mouse (Cadwell et al., 2015).
The teams used similar experimental approaches: whole-cell patch-clamp recordings on brain slices were followed by backfilling of the recorded cells for morphological characterization, aspiration of the soma and single-cell RNA-seq of the aspirate. Although Patch-seq resulted in fewer detected transcripts than obtained with conventional single-cell RNA-seq approaches, both teams resolved thousands of genes and found strong correlations between functional and molecular properties. Both teams were able to explain a variety of electrophysiological properties on the basis of differential gene expression.

Linnarsson and Harkany's team used Patch-seq to refine the classification of interneurons in the L1 layer of the mouse somatosensory cortex by mapping the data onto an existing molecular classification obtained from previous unbiased sequencing of neurons from the same brain region. Sandberg, Tolias and their team did not attempt to fur- ther subdivide the two classes of neurons in the L1 layer of the neocortex, but they applied the Patch-seq technology in vivo in anesthetized mice and obtained similar results, albeit at lower efficiency.

The Patch-seq technology will be useful for analyzing cellular heterogeneity in the brain and for addressing the molecular mechanisms of cell-type diversity. Linking neuronal function at the levels of gene expression and electrophysiology in the same cell is more powerful than addressing neuronal function by averaging over populations of neurons. Nina Vogt

RESEARCH PAPERS

Fuzik, J. et al. Integration of electrophysiological recordings with single-cell RNA-seq data identifies neuronal subtypes. Nat. Biotechnol. doi:10.1038/nbt.3443 (21 December 2015).

Cadwell, C.R. et al. Electrophysiological, transcriptomic and morphological profiling of single neurons using Patch-seq. Nat. Biotechnol. doi:10.1038/nbt.3445 (21 December 2015). 